\title{
Ambient Artificial Intelligence
}

\author{
Shashank Agnihotri \\ B.E. Computer Engineering \\ VES Institute of Technology \\ Mumbai- 400074
}

\author{
Anshul Agarwal \\ B.E. Computer Engineering \\ VES Institute of Technology \\ Mumbai- 400074
}

\begin{abstract}
The paper presents an integrated, automated and wireless system concept for the human intelligence environment domain based on the technique called ambient artificial intelligence (AAI). Ambient Intelligence system is that which is embedded in an environment, enhancing complex and manual life to simple and automatic life. This technology in an environment is challenging to various algorithm that are being used with respect to human behavior and human life. Development of such intelligent and smart environment is dependent on the adaptive nature, flexibility, robust parameters of such intelligent system which are mainly forecasted on certain factors like reasoning, decision making and acting. The details of new emerging technology, ambient artificial intelligence, its working, architecture and various technologies used to build such efficient system. ${ }^{[1]}$
\end{abstract}

\section{General Terms}

Ambient, population, mobile application, trending, syntactic analysis, natural language processing.

\section{Keywords}

Informative environment.

Environment, Reasoning, Ubiquitous

\section{INTRODUCTION}

Ambient- relating to the immediate surroundings of something. The development in computer science is continually evolving around the world on the fast track. Amongst this, one of the major factor is Ambient Intelligence. The term 'Ambient' is defined as 'to cover' something from all the corners. AAI is an upcoming technology which will enhance and ease one's day to day life, which then help one to improve discipline and quality of life. AAI has the ability to serve the environment, which is intelligent and able to adapt the human's changing behaviour and help to live luxurious life. This is adaptable to the humans and help in their needs and goals by consolidating sensing capabilities, reasoning mechanisms, digital content. It is also able to recognize one's presence, equip us with information according to one's immediate needs. ${ }^{[2]}$

AAI technology is mainly focused on an interface which aids and helps human beings in their daily activity. It is implemented through a simple mobile application installed in smartphones. This technology requires quick access to servers and an extensive interface between the user and the application.

There are two issues created due to evolution of mankind. The issues are:

In this 21 st century the whole world is connected and any news in any part of the world, directly or indirectly, makes a difference for rest of the world as well, thus being informed of everything in one's surrounding, in one's ambience is very important. But how is this achieved?

With the population across the globe growing in education and literacy every day, and people being judgemental of others even based on small things like shoe colour or grammar, it becomes very important to be presentable, correct and confident. But how is this achieved?

Instead of blaming the evolution of mankind for the problems caused, it is essential to come up with solutions to the problems caused so that mankind has a healthy environment to live in. The solution to both these broad problems and many other subsidiary problems that follow has been discussed in this paper.

The paper proposes an extensive mobile application as a solution to above issues. This mobile application will have a keyboard and even a background application which will continuously collect data from every user and send to the local servers for processing and storage. Then this application will even have an interface to inform user of trends round the globe and many other features like alarms, notifications, keyboard prompts etc.

\section{BASIC CONCEPT}

AAI is comprises of three basic modules, that are, fetching information, validation of information and delivering correct and concerned information. All three of these modules are interrelated with each other and are defined as the backbone of AAI.

\section{Fetching Information}

Fetching information is the primary step of AAI. It is responsible to collect information from each and every user. This is achieved by two features of a mobile application. One feature is a keyboard which keeps a track of every word and emoticon used by the user, while the other is scanning software which scans all data including images and videos on a user's device. All the information collected from the user is sent to local server with the timestamp of that information and the count of occurrence of particular information in cases of repentances if any.

\section{Validation and Processing of Information}

The information is being sent by multiple users to the local servers every moment. This information sent is processed and divided into two categories, which are, keyboard input and user data.

Keyboard input will consist of words, digits and emoticons. Each word coming in would be processed and categorized into categories like "Trend", "Travel", "Work", "Family" etc. One piece of information might be categorized into more than one category. Then this, just arrived piece of information is sent to storage for saving user data as well as verified for correct grammar and usage of that language. This verification 
is done with the already present and established libraries. In case of clashes, the information with more weightage is given more preference. This weightage would be discussed later. If the incoming information has more weightage than the stored information or rules then the stored information is replaced, but if the stored rules or information has more weightage than the user is notified of the corrections immediately by hints on the keyboard.

User data is basically all the browser history, contacts, videos and photos in scanned in the user's device. It contains vital information about the user. This information is processed by machine learning and artificial intelligence algorithms which extract information regarding various issues and topics related to the user and categorize them in the earlier mentioned categories. This information is stored in the local servers and is sent as an update to the central server.

Both central and local server scan through information gathered from other users and information already present in the storage and find something relevant which will help the user in their work. This information collected is then sent to the user as a notification or in the assistant interface of the application. Example: If the application finds a photo of a beach in the user's device and sends it to the local server. The local server scans the image, extracts information from it and sends it the central server and also to its own memory for processing. The servers find that the image is of Hawaii, then the servers finds all the information it has or can acquire on Hawaii like the travel rates from user's current location and frequently visited locations in Hawaii, the prices of different hotels in Hawaii, the places to visit in Hawaii, etc. All this information is sent to the user's device.

\section{Delivering Correct And Concerned Information}

All the information collected and processed in the previous module now needs to be delivered accurately to the user, such that, the system does not bug or interrupt the user unnecessarily. It delivers the information in such a manner that the user is able to make a note of it.

The corrections to the typing of the user can be achieved by highlighting or underlining the incorrectly typed words by the user in the typing window, before the user can send it, and suggesting the corrections in a narrow division just above the keyboard or as a drop down menu which appears on tapping the highlighted word. For further simplicity and ease in future the often misspelled words can be saved on the user's device itself and auto-corrected by the keyboard application. The keyboard application with the help of machine learning algorithms will learn the way the user types, talks. The specific replies the user gives to specific people or group of people and over a period of time generate a pattern and forecast the user's reply to a specific message, and hint the user with these replies. In such cases, the user would not have to type his message but just tap on the word they want next, or the sentence needed next as and when prompted by the system.

The information sent to the user other than typing corrections is used to serve a vital role of the application. This information can be sent as a notification on the user's device or as a separate section in the Ambient Assistant window. The user would be provided with a notepad where they can save the date for future reference. Also the frequently used data would be stored separately by the device for faster references in the future.

\section{CHARACTERISTICS OF AAI}

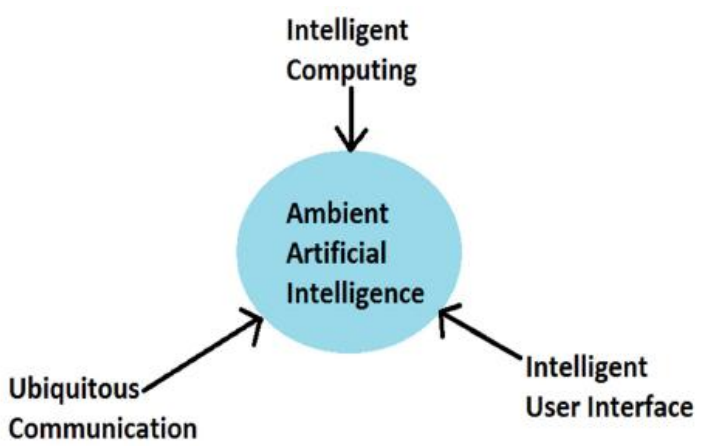

Fig 1: Characteristics of AAI

AAI builds on three most recent technologies that are:

1. Intelligent Computing

2. Ubiquitous Communication

3. Intelligent User Interface

\section{Intelligent Computing}

Intelligent Computing is the most important characteristic of this application. The machines at the servers, both central and local are loaded with machine learning algorithms and artificial intelligence algorithms. The machine learning algorithms are used in classification problems and training the servers and devices with user inputs. The artificial intelligence algorithms are used in building user profiles and forming patterns and character sketch of each user and deciding when and where which user will need what information or assistance and making that possible. ${ }^{[3]}$

For Example: If the user is travelling from Mumbai to Delhi, and the user is a resident of Mumbai, then the user will need air tickets to Delhi, but the user usually travels cheap, then the system will prompt the user with air tickets priced low, and available when the user plans to go to Delhi, the system will prompt the user with cab services available in Delhi, and hotels to stay in Delhi. The most striking feature about this would be that the user would not need to instruct the system to do all this, although if the user wishes to, the user can, but if not so, the system will automatically learn that the user is planning a trip to Delhi, by his messages, emails and other media. The system will suggest cabs and hotels according to the salary and the likes and dislikes of the user, and also the proximity to the destination the user intends to visit, which again the system will learn on its own or can be specifically instructed.

\section{Ubiquitous Communication}

The word ubiquitous is defined as "existing everywhere and anytime", "scattered in an environment", and widespread area". While using this term technically implies that technology is present everywhere. In other words, humans are being monitored by various technologies itself.

Ubiquitous communication is the next logical step after setting up all devices that are used for intelligent computing. The basic aim for ubiquitous communication is to communicate between human and devices or between any devices via the local or central servers.

For Example: If the MLA of a certain region announces a new scheme for his region then all the users in that region, that is only the concerned parties, will get an update about it, not users in the other regions to which the information does not 
concern. But if the PM of a nation announces a policy for the nation then all the users of that nation and the users of the nations that the policy will affect will get an update of the same.

\section{Intelligent User Interface}

Intelligent User Interface is multi-media model way to interact with the human and computer. The interaction enables the users of the AAI system to be informed of their surroundings and control and interact with the application to get desired output in way of voice and gestures, personalized way by preference and context and physical interaction. These interactions would be serviced by the Ambient Artificial Intelligence Assistant, or just the Ambient Assistant.

\section{ARCHITECTURE OF AAI}

\section{OUTSIDE WORLD}

\section{APPLICATION USER INTERFACE}

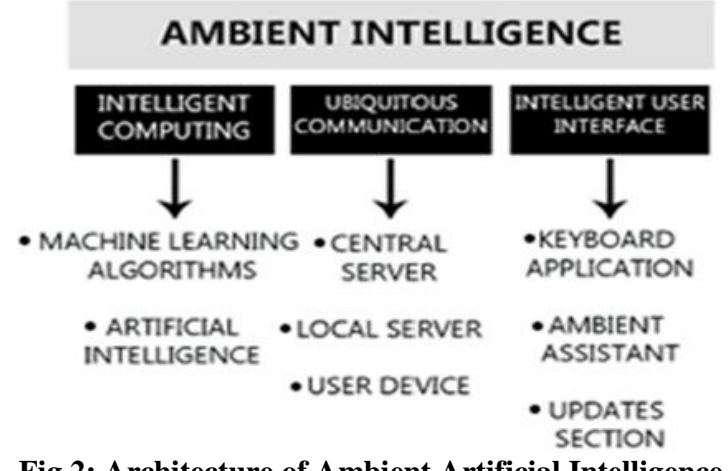

Fig 2: Architecture of Ambient Artificial Intelligence

\section{Machine Learning Algorithms}

As the user types using the keyboard each word is being sent to the local server. The local server has learning algorithms which learns the way particular user types, their patterns and the interests of the users by scanning and processing of the images, contacts, mails, videos and other information with the user. This, what the system learns is sent back to the user's device and over a period of time the user's device is trained enough to behave like the user itself. It will start prompting exactly what the user needs, exactly what the user intends to type and exactly what the user needs to be informed of.

\section{Artificial Intelligence}

Artificial intelligence is used by the system in understanding user patterns and doing tasks for the user which the user intends to do or would intend to do.

For Example: The system learns that the user pays his electricity bill on the 4th of every month from his 'freecharge' account. So the system will automatically scan the electricity bill, extract the amount of bill to be paid, check if the user has already paid the bill, if not then pay the bill and notify the user that the electricity bill for that month has been serviced by the system already.

\section{Central Server}

If this network or system is compared to the nervous system of our body, then the user devices are sense organs, local servers are neurons and spinal cord, while the central server is the brain.
The central server is the most important component of the entire project. For correction of words typed or grammar checking of what the user is typing the central server is initially trained in grammar of all the languages in the world and the priority for different regions is coded in. Now when the central server is passed on information from the local server then the central server compares that information with the already present rules and information it has. If similar information does not exist then the central server adds that information or rule to its storage with the priority level of the region or local server it got the information from and passes on the rule or information to all the local servers, and on each repetition of that information or word its weightage increases, but if the information exists then the central server compares the two information, if they are similar then there is no problems and central server notifies the local server that the information was correct and is verified, but if there is a clash between the two information then the information with higher weightage gets preference and is considered correct by the central server and passed on to all the local servers.

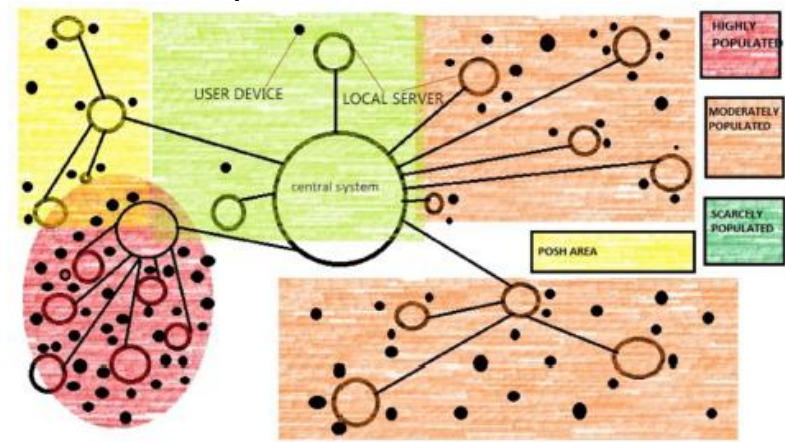

Fig 3: Distributed Database System to implement AAI.

Each location or region has a set priority or weightage, and all the local servers lying in that region by default are initialized to that weightage. This weightage is useful in case of clashes in the present rules or grammar and the one, the user has just used. The weightage of a local server exponentially increases with the number of connected lower local servers to it.

The posh area has the highest weightage assuming that people their will mostly type correct content, followed by highly populated areas which has second highest weightage followed by moderately populated areas and then lastly the scarcely populated areas.

The central server has another responsibility, to decide what content is trending in which region. Whether a news or trend is international, national, state level, regional or local. If all the local servers are pinging the central system around the same time, with the same keywords or information then the news or trend is worldwide, but if the local servers of only particular areas are pinging the central server with a information or similar keywords then the information, news or trend is a hot topic in only those areas. The central server accordingly takes a decision call and updates the required local servers with what's trending in their areas and what may trend.

\section{Local Servers}

The local servers are the backbone of this architecture. They form a direct link between the user devices and the central system. All the user devices are connected to the nearest local servers. The local servers get information from the users' devices and process it and sends it to the central system. If it is a keyboard input then it is verified by the local server if similar information has been encountered by the local server 
in the past, if not then it is sent to the central server the verified information and rule is saved in all the local server for further reference so that next time for a similar verification the central server is not needed, unless there's a clash. In case of a clash between the local server and the user device input, the central server is sent the information and the central server deals with it and sends the correct output to the local server, which saves it for future reference and even updates the user about it. For each and every user input, there is verification and suggestion by the local server.

In case of trends and news, if a local server is hit repeatedly with the same keywords then it sends a request to the central server, to call this information a trend, the central server takes the decision call and replies to the local server with the title for that trend, meaning if it is local, state, international, national, etc or not a trend at all. The local server propagates to the user devices connected to it accordingly.

\section{User Device}

AAI has a Distributed Database Architecture, of which User's devices are the lowest but the most important module. All the information regarding a user is gathered at this point and then sent to the local servers, and the information from the local servers comes here for notifying the user. All the information stored in any form of media or text is recorded and sent to the local servers.

\section{Keyboard Application}

This is the place from where the user's device gets its data which it sends to the local servers. Each and every letter, digit, emoticon the user types, the pattern in which the user types and the reason for which the user types, the purpose of that information and recipient, the typing speed, everything is recorded with the time stamp and stored in the user device to be sent to the local servers. This is the most important source of information for our system, as the Ambient Assistant and Updates Section gets major chunk of its information from this section.

\section{Ambient Assistant}

The Ambient Assistant gets its data from the keyboard and all the recorded information fetched, processed and stored in the user's devices. This assistant helps and notifies user of its needs. This assistant will automatically pay the user's' bills, book a cab for the user, show way to home from any location on maps, show route and modes of transport to travel from a particular location to any other location, book flight tickets for the user, chat with someone in place of the user, do literally anything, without the user instructing the assistant to do, by just confirming the assistant's request to do so. The assistant will even have semantic analysis. This analysis will enable the application to analyze the user's mood and help the user with the necessities like prompt jokes if the user is feeling low, dial friends or support system if the user shows suicidal tendencies.

\section{Updates Section}

This section will update the user with current news and trends around, with everything sorted in categories like International, regional etc. Not just this, the updates section would even update the users of the social life of their friends, let the user know of any particular event the user wants to know of, without the user asking the system to do, but by just getting updates from the user device.
If the topic in the Updates section is a hot topic with many users giving their views on it then their views would be recorded and these views would appear in the updates section with the topics, though the name of the users would not be disclosed unless the user, giving the view, wants it to be disclosed. By this feature a user will the know the common notion on a topic, even statistical analysis would appear.

For Example: If the topic is Demonetization of Bank notes in India, then statistical analysis would appear on how many favor it and how many are against it based on the views recorded by the user's devices, local servers and central servers.

\section{SCENARIOS}

\section{AAI As A Spelling And Grammar Checker}

When a user is typing anything on the keyboard, each and every word is being verified by the stored rules and words in the user device from past usage, if in the user device that word or rule is missing, then it is verified by the local server, if it's missing in the local server as well then it is verified by the central server, and on the way back in the hierarchy the new encounter is saved. Same hierarchy is followed in case of clashes. Now, as the user types, simultaneously, based on previous experience and user's character sketch formed by the system, the user would be getting prompts of words, prompts of sentences, internet links, emoticons, numbers, or a combination of all of these. So now the user does not need to type what it wants to send, it can just tap the option it finds most suitable and send that, or the user can even voice and gesture control the options. The user would have the option to edit the automatically generated response as well.

\section{AAI As An Assistant}

Suppose a user has received a message on WhatsApp where a relative has asked him in how much time would the user meet him, and the user replies that he will meet his relative in five minutes, and that very moment the user gets an email saying that he has a job interview in 1 hour at a location 15 minutes away from his current location and 10 minutes away from his relative's place. The user's device had earlier learnt that the user has a 15 minutes work with his relative and the user is a family man, but is also very particular about work. The user's device from user's photos and bills has also learnt that the user has no vehicle, has only one suit and he has given that suit to the laundry.

The Ambient Assistant would immediately send a message to the laundry that the user is coming to pick up his suit in 2 minutes so he should keep it ready, the assistant would book a cab from user's location to his relative's location via the laundry, and book another cab, after the calculated time interval, counting the traffic, cab arrival time, and time taken by the user, from the relative's location to the location of the interview. The assistant would save an alarm to remind the user to take all the necessary documents for the interview and even remind the user of the work he has with his relative. After the user picks up his suit and meets his relative, the assistant would save another alarm to notify the user that he is getting late for his scheduled interview.

\section{AAI As An Updater}

Suppose the Prime Minister of India announces a new scheme for the nation, then all the users would be typing this and conversing about this very topic in India. The user's device will ping the local server saying that this is the hot topic. 
Every local server will ping the central server saying that this is the hot topic in their respective zones or regions. The central server analysis and understands that this is the hot topic in India as everyone in India is discussing about this. However no one in Europe or Africa is talking about this, but this topic concerns Northern American Nations, so the central server will revert to the local servers in India and North America informing that this is the hot topic trending and will attach to it the views expressed by various users. This will even sync with the keyboard, as if a user's views seem to match to any other user around the globe, based on past experience and scenarios of the user, then this user while typing his views to a friend on chat or on mail to someone, would directly be prompted the other user's already typed view with modifications according to the user, thus reducing his efforts.

\section{IMPLEMENTATION}

For implementing this project the basic needs are Distributed Database System, server farms, high speed supercomputers and very high speed internet connection with negligible latency.

Other than that, project uses Machine Learning and Artificial Intelligence algorithms which play major role in the entire project. For classification problems we can employ Neural Networks in deep learning.

The application needs artificial intelligence to parse messages sent and received by the user. Natural Language Processing (NLP) algorithms can be used for this purpose. Naive Bayes classifier is used very frequently in NLP, much more so that in most other machine learning-related fields.

\section{LIMITATIONS}

This project has a few limitations. Some of them being:

\section{Over reliance on AI}

If we rely on machines to do almost everything for us we become dependent, so they have the potential to ruin our lives if something were to go wrong.

\section{Misuse}

There is no doubt that this level of technology in the wrong hands can cause mass destruction.

\section{Need of Users}

For this project to be successful, large number of people need to use it regularly.

\section{Privacy}

Private information of the users needs to be compromised.

\section{FUTURE SCOPE}

The current news feed sections available tend to provide redundant and news not concerning the user. This causes unnecessary traffic on channels, traffic that could have been avoided. The Ambient Artificial Intelligence's scope would be to be the most optimal news delivering system.

By incorporating Lifelong Learning ${ }^{[4]}$ in the AAI system, the system can be made to think and make decisions like humans. The way human beings learn, similarly the system will learn and behave accordingly.

\section{CONCLUSION}

Giving insufficient attention to the challenges posed by Ambient Artificial Intelligence technologies bears the risk that the negative side effects will overshadow the envisaged benefits. For most companies, the key motivation for investing in new technologies is usually to increase efficiency in order to maximize profits. But by solely focusing on performance affects, other aspects of knowledge work are usually neglected. Besides the performance, which is often used, as it is relatively easy measure, a variety of other factors are also affected through the implementation of new technologies. While future office environments will be implemented using a variety of new technologies, the design of systems and applications should not be bound or driven by these technologies. Instead of being determined by software and hardware possibilities and constraints, the design needs to be driven by humanistic needs and concerns. The design of smart environments will have a considerable impact on the way people work and interact in these environments. The possibilities that arise from Ambient Artificial Intelligence will not only influence organizational processes, but also the way daily work is organized.

\section{REFERENCES}

[1] Manish Gangwani, Nitin Jotwani, Manisha Galani, Anjali Yeole. Ambient Intelligence. Mumbai University.

[2] Werner Weber, Jan Rabaey, Emile H.L. Aarts, “Ambient Intelligence” Springer Science \& Business Media.

[3] "Affective Computing" MIT Technical Report \#321 (Abstract), 1995

[4] Chen, Zhiyuan, Nianzu Ma, and Bing Liu. "Lifelong Learning for Sentiment Classification." ACL (2). 2015. 